\title{
Flexible and Environment-friendly Regenerated Cellulose/MoS2 Nanosheet Nanogenerators with high Piezoelectricity and Output Performance
}

\section{Siling Chen}

Wuhan University of Technology School of Materials Science and Engineering

Jilong Li

Wuhan University of Technology School of Materials Science and Engineering

Yiheng Song

Wuhan University of Technology School of Materials Science and Engineering

Quanling Yang ( $\sim$ yangql@whut.edu.cn )

Wuhan University of Technology https://orcid.org/0000-0002-7013-419X

\section{Zhuqun Shi}

Wuhan University of Technology School of Materials Science and Engineering

\section{Chuanxi Xiong}

Wuhan University of Technology School of Materials Science and Engineering

\section{Original Research}

Keywords: Piezoelectric, Cellulose, MoS2 nanosheet, Nanogenerator

Posted Date: February 12th, 2021

DOl: https://doi.org/10.21203/rs.3.rs-184100/v1

License: (9) (i) This work is licensed under a Creative Commons Attribution 4.0 International License. Read Full License 


\section{Abstract}

Flexible piezoelectric nanogenerators for energy harvesting are getting more and more attention nowadays by converting the mechanical energy to electric energy. Here, an environment-friendly piezoelectric nanogenerator based on the regenerated cellulose (RC)/MoS 2 nanosheet nanocomposite successfully exhibited a relative high output voltage of $2 \mathrm{~V}$ and current of $150 \mathrm{nA}$ under slight press which were 5 and 7.5 times higher than those of the neat RC film, i.e. $0.4 \mathrm{~V}$ and $20 \mathrm{nA}$, respectively. In particular, the $\mathrm{MoS}_{2}$ nanosheets were obtained through a simple, facile and low-cost pathway by mechanical exfoliation in triethanolamine. The nanocomposite film with $\mathrm{MoS}_{2}$ nanosheet content of $4 \%$ exhibited a high piezoelectric constant $\left(d_{33}\right)$ of $19 \mathrm{pC} / \mathrm{N}$, which was 6.3 times higher than that of the neat $\mathrm{RC}$ film (i.e. $3 \mathrm{pC} / \mathrm{N}$ ). Thus, the $\mathrm{RC} / \mathrm{MoS}_{2}$ piezoelectric nanogenerator has great potential applications in the fields of energy harvester, sensors and is of great significance to environment protection.

\section{Highlights}

1. $\mathrm{MoS}_{2}$ nanosheets were prepared by facile exfoliation in triethanolamine.

2. The $\mathrm{MoS}_{2}$ nanosheets greatly improved the piezoelectricity of cellulose.

3. The nanogenerator is flexible and environment-friendly with high output performance.

\section{Introduction}

With the consuming of fossil energy and deteriorating of environment, the quest for clean and renewable energy has become an urgent challenge (Goldemberg 2007; Wang et al. 2019). Energy harvesting technology is one of the potential methods in energy utilization. There are many sustainable energy sources that can be used in energy harvesting such as solar energy, wind energy (Fan et al. 2016). Piezoelectric materials have attracted more and more attention as a new pathway that can convert mechanical energy in ambient environment to electric energy and is promising to realize energy generation (Sappati and Bhadra 2018; Park et al. 2014; Zhao et al. 2020). In 2006, Wang et al. (2016) developed a piezoelectric nanogenerator made of $\mathrm{ZnO}$ nanowire arrays for the first time, which could harvest mechanical energy and convert to electric energy. There is a potential difference when stress is applied to piezoelectric materials, which is called positive piezoelectric effect. Besides, the converse piezoelectric effect is that the material deforms under electric stimulation (Wei et al. 2018). Energy harvesting technology inspired by piezoelectric effect can be used in nanogenerator (Ye et al. 2019), selfpowered sensor and so on (Fang et al. 2019). The devices could convert mechanical energy from human daily life such as walking, bending at anytime and anywhere to achieve the purpose of energy utilization (Yang et al. 2016; Xu et al. 2010). Furthermore, when this kind of self-driven sensor used in vivo environment as medical device (Zhao et al. 2020), the problem of battery replacement can be avoided because it can extract biochemical energy like heartbeat (Wang and Wu 2012; Vivekananthan et al. 2018). 
So far, some types of piezoelectric materials have been studied (Chen et al. 2020). Organic piezoelectric polymers such as polyvinylidene fluoride (PVDF) and their copolymer polyvinylidene fluoridetrifluoroethylene P(VDF-TrFE) (Li et al. 2020; Yang et al. 2020; Lovinger 1983), are flexible and light weight but of low piezoelectric coefficient. On the other hand, inorganic piezoelectric ceramics such as lead zirconate titanate piezoelectric ceramics (PZT) (Park et al. 2017), potassium sodium niobate (KNN) and barium titanate $\mathrm{BaTiO}_{3}$ ( $\mathrm{Lv}$ et al. 2020; Koda and Sodano 2014) exhibit superior piezoelectric properties but high brittleness, poor toughness and some high toxicity, thus their application is limited (Shi et al. 2019). For practical application, the polymer matrix usually composites with inorganic piezoelectric ceramic to prepare the flexible nanogenerator with optimized piezoelectricity (Wu et al. 2019).

Furthermore, most synthetic polymers, e.g. PVDF and P(VDF-TrFE), are not biodegradable and they would cause severe environmental problems after usage. Moreover, they are not renewable and biocompatible. Therefore, seeking for renewable, biodegradable, and biocompatible natural polymer for piezoelectric nanogenerator is urgent. Cellulose is the most abundant and widespread natural polymer in the nature (Kim et al. 2019; Wang et al. 2014; Song et al. 2021). It is a hydrophilic glucan biopolymer consists of a linear chain of two anhydroglucose rings joined via a $\beta-1,4$ glycosidic linkage (Pandey et al. 2010; Klemm et al. 1998). Compared with many synthetic polymers, cellulose has many excellent features: renewability, biocompatibility, biodegradability, high strength, and low thermal expansion (Tayeb 2019; Mishra et al. 2019). They have been explored for applications in electronics and functional devices (Soba et al. 2016; Chen et al. 2020; Toroń et al. 2018; Zhai et al. 2015; Yin et al. 2020). However, cellulose cannot be dissolved in common solvent or melt because of its strong intra and inter hydrogen bonding (Zhang et al. 2007). Zhang et al found that cellulose could be dissolved in $\mathrm{NaOH} /$ urea aqueous solution under low temperature (Cai and Zhang 2005). Cellulose films could be obtained through this non-toxic and low-cost solvent system after regeneration (Qi et al. 2009).

Recently, as a novel high-performance piezoelectric material, monolayer or few-layer molybdenum disulfide $\left(\mathrm{MoS}_{2}\right)$ has attracted great attention (Sohn et al. 2019; Wu et al. 2021). Sahatiya et al. facricated a nanogenerator which employ both piezoelectricity and triboelectricity based on $\mathrm{MoS}_{2}$, cellulose and PVDF (Sahatiya et al. 2018). MoS $_{2}$ has layered structure in which a molybdenum plane is sandwiched between two sulfide planes connected by Van der Waals force, while the molybdenum and sulfide atom are covalently bonded (Acharya et al. 2018). Zhou et al. found that $\mathrm{MoS}_{2}$ with odd-number layers could generate high piezoelectric voltage and current under stress, which increased with the number of layers decreased. $\mathrm{MoS}_{2}$ with odd number has strong piezoelectric effect because of the asymmetry of positive and negative charge resulting from the deformation under external forces. On the contrary, $\mathrm{MoS}_{2}$ with even number did not exhibit piezoelectricity owing to the presence of a projected inversion symmetry (Zhou et al. 2016), the positive and negative charge cancel each other out. There are several methods to obtain $\mathrm{MoS}_{2}$ nanosheet, such as chemical vapor deposition (CVD) and mechanical delamination (Krishnamoorthy et al. 2016), but these methods are of low yield and efficiency. However, liquid exfoliation is a simple way to prepare 2D materials (Niu et al. 2016). Recently, we found that monolayer 
or few-layer $\mathrm{MoS}_{2}$ nanosheets could be obtained by mechanically stirring in triethanolamine, which is effective and low cost (Chen et al. 2017).

Here, we constructed a flexible, lead-free and biocompatible piezoelectric nanogenerator by compositing of $\mathrm{MoS}_{2}$ nanosheets and cellulose molecules for energy harvesting. $\mathrm{MoS}_{2}$ nanosheets were one-step exfoliated by triethanolamine, and cellulose was dissolved in $\mathrm{NaOH} / \mathrm{urea} / \mathrm{H}_{2} \mathrm{O}$ solution. $\mathrm{MoS}_{2} /$ cellulose nanocomposite films were obtained by blending exfoliated $\mathrm{MoS}_{2}$ nanosheets and cellulose, followed by regeneration, washing, and natural drying. Then, they were used to fabricate the piezoelectric nanogenerator with $\mathrm{Al}$ foil as the electrode, $\mathrm{Cu}$ wire as the connecting wire, and polyimide (PI) as the encapsulation layer. The piezoelectricity, output voltage and current of the nanogenerator under press were studied in details.

\section{Materials And Methods}

\subsection{Materials}

Cellulose sample (cotton linters pulp, a-cellulose content of more than 95\%) with a weight-average molecular weight of $10 \times 104 \mathrm{~g} / \mathrm{mol}$ was provided by Hubei Chemical Fiber Co., Ltd. (Xiangyang, China). Molybdenum disulfide ( $\mathrm{MoS}_{2}, 98 \%$ ) was bought from Sigma-Aldrich Corporation (Saint Louis, USA). Triethanolamine $\left(\mathrm{C}_{6} \mathrm{H}_{15} \mathrm{NO}_{3}, 98 \%\right)$, sodium hydroxide $(\mathrm{NaOH}, \mathrm{AR})$, urea (AR) and other chemical reagents were purchased by Shanghai Aladdin Industrial Corporation, China.

\subsection{Exfoliation of $\mathrm{MoS}_{2}$ nanosheets}

$0.5 \mathrm{~g} \mathrm{MoS}_{2}$ was added to $50 \mathrm{ml}$ triethanolamine and stirred for $8 \mathrm{~h}$ at $1000 \mathrm{r} / \mathrm{min}$, followed by centrifugation for 30 minutes at $4500 \mathrm{r} / \mathrm{min}$. After washing the supernatant with deionized water and filtering several times to remove triethanolamine, the obtained product was dispersed in deionized water followed by freeze-drying to prepare the $\mathrm{MoS}_{2}$ nanosheets.

\subsection{Preparation of $\mathrm{RC} / \mathrm{MoS}_{2}$ films}

The $\mathrm{NaOH}$, urea and deionized water were weighed at mass ratio of 7: 12: 81 and well mixed to make a $\mathrm{NaOH} /$ urea/ $\mathrm{H}_{2} \mathrm{O}$ solution. The obtained $\mathrm{MoS}_{2}$ nanosheets were homogeneously dispersed in the $\mathrm{NaOH} / \mathrm{urea} / \mathrm{H}_{2} \mathrm{O}$ solution by sonication and cooled to $-13^{\circ} \mathrm{C}$. Then a desired amount of cellulose was dissolved in the above dispersion to prepare the composite solution. After removing bubbles by centrifugation, the composite hydrogels were prepared by casting the composite solution on the glass plate and then immersing in dilute sulphuric acid (5\%) for 5 minutes. The regenerated cellulose (RC)/MoS ${ }_{2}$ nanosheet composite films with thickness of 20-30 $\mu \mathrm{m}$ were finally obtained after washing and immersing the composite hydrogels in water to remove residual reagent, followed by air-drying. These obtained films were denoted as RC, RC/ $\mathrm{MoS}_{2} 1, \mathrm{RC} / \mathrm{MoS}_{2} 2, \mathrm{RC} / \mathrm{MoS}_{2} 4$, and RC/ $\mathrm{MoS}_{2} 8$, 
respectively, with incorporation of $0 \mathrm{wt} \%, 1 \mathrm{wt} \%, 2 \mathrm{wt} \%, 4 \mathrm{wt} \%$, and $8 \mathrm{wt} \% \mathrm{MoS}_{2}$ nanosheets in the composites.

\subsection{Preparation of RC/MoS 2 nanogenerators}

The above obtained films were polarized under an electric field of $15 \mathrm{MV} / \mathrm{m}$ in silicone oil bath for $40 \mathrm{~min}$ at room temperature. Then for construction of nanogenerators, these films were sandwiched between Al foil as electrode, copper wires were connected to the Al foils as connecting wires, and polyimide (PI) as encapsulation layer to protect nanogenerators from the influence of external charge.

\subsection{Characterization}

The morphology and microstructure of the sample was characterized by scanning electron microscope (SEM) (JSM-IT300, JEOL, Japan) after the sample was fractured in liquid nitrogen. Fourier-transform infrared (FTIR) spectra of the samples were examined by FTIR spectrometer (Nicolet6700, USA). The Xray diffraction (XRD) (D8 Advance, AXS, German) analysis was recorded with Cu Ka source radiation. The mechanical property was carried out by universal tester (RGM-4100, Geger, China), the samples were selected from the uniform part of the films and cut into $30 \mathrm{~mm} \cdot 5 \mathrm{~mm}$, and the stretching speed was set at $2 \mathrm{~mm} / \mathrm{min}$. Thermal stability test (TGA) was measured by thermal analyzer (STA499C, Netzsch, Germany) in the nitrogen atmosphere and the temperature with the range of 0 to $100{ }^{\circ} \mathrm{C}$ at an increase rate of $10^{\circ} \mathrm{C} / \mathrm{min}$. Dielectric constant and loss was tested through Agilent equipment (HIOKI3532-50, HIOKI, Japan) in the frequency from 100 to $1 \mathrm{M} \mathrm{Hz}$. The piezoelectric coefficient $d_{33}$ was obtained via quasi-static $d_{33}$ tester (ZJ-3A, Chinese academy of sciences) after polarization. The piezoelectric performance including output voltage and current of the nanogenerator was measured by electrochemical workstation under a slight press about $0.88 \mathrm{kPa}(\mathrm{CHI} 660 \mathrm{E}$, Shanghai, China).

\section{Result And Discussion}

\subsection{Structure of $\mathrm{RC} / \mathrm{MoS}_{2}$ nanocomposites}

Figure 1 shows the fabrication process of $\mathrm{RC} / \mathrm{MoS}_{2}$ film and construction of piezoelectric nanogenerator. The film was fabricated through blending, casting, regeneration, air-drying and the process is relatively simple and environmentally friendly. Besides, the obtained device has superior flexibility so that it can be used in many fields, e.g. detection of human physical signals.

Figure 2 shows SEM images of the cross-sections of the RC and RC-MoS 4 films. The layered structure of the RC film which comprised of uniform and compact fibrils was observed in Fig. 2(a, b). Figure 2(c, d) reveal the morphology of RC-MoS 44 film, where with the incorporation of $\mathrm{MoS}_{2}$ nanosheets, more obvious and compact layered structure was clearly observed. Moreover, there is no obvious aggregation and defect in the RC-MoS ${ }_{2}$ composite film, and it shows good compatibility between $\mathrm{MoS}_{2}$ nanosheets and cellulose molecules, probably owing to that the intercalation of cellulose molecules in $\mathrm{MoS}_{2}$ 
nanosheets and formation of hydrogen bonding between them provide hindrance to prevent $\mathrm{MoS}_{2}$ nanosheet from stacking.

To further clarify the hydrogen bonding between cellulose molecules and $\mathrm{MoS}_{2}$ nanosheets, and also

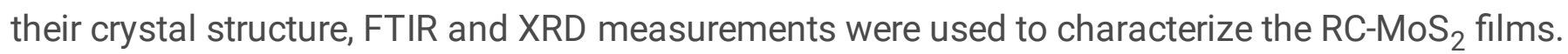
Figure 3 is FTIR spectra of RC-MoS ${ }_{2}$ films with different filler content which was recorded from 4000 to $400 \mathrm{~cm}^{-1}$. The broad absorption band around $3448 \mathrm{~cm}^{-1}$ is corresponding to stretching vibration of hydroxyl groups of cellulose. Compared with RC film, this broad band of RC-MoS ${ }_{2}$ film shifted to lower wavenumbers which indicated that with the addition of $\mathrm{MoS}_{2}$, original hydrogen bonding in cellulose molecules were broken and new stronger hydrogen bondings between cellulose molecules and $\mathrm{MoS}_{2}$ nanosheets were formed (Raghunathan et al. 2017). Besides, the peaks at $1418 \mathrm{~cm}^{-1}$ and $1017 \mathrm{~cm}^{-1}$ represent the bending vibration of $\mathrm{C}-\mathrm{H}$ and stretching vibration of $\mathrm{C}-\mathrm{O}-\mathrm{C}$ group.

Figure $\mathrm{S} 1$ shows the XRD patterns of the RC, $\mathrm{MoS}_{2}$, and RC-MoS 2 composite films. The XRD pattern of the bulk $\mathrm{MoS}_{2}$ displays a (002) plane at $2 \theta=14.4^{\circ}$ which is feature peak of $\mathrm{MoS}_{2}$. The $d$-spacing of the (002) plane of bulk $\mathrm{MoS}_{2}$ was calculated to be $0.61 \mathrm{~nm}$ using the Bragg equation. On the other hand, the $\mathrm{RC}$ film exhibits three $2 \theta$ diffraction peaks at $12^{\circ}, 20^{\circ}$, and $23^{\circ}$, owing to the $(1-10),(110)$ and $(020)$ crystal planes of cellulose II, respectively (French 2014, 2020). The relative higher intensity of the $2 \theta$ diffraction peaks at $12^{\circ}$ indicates the preferred orientation of the (1-10) plane parallel to the film surface, probably because of the regeneration of cellulose in the aqueous system and the film drying process (Isobe et al. 2011; Yang et al. 2015; Yin et al. 2020). For the RC-MoS ${ }_{2}$ composite films, the feature peaks of XRD patterns are almost corresponding to the primary feature peaks of $\mathrm{MoS}_{2}$ and RC. The $d$-spacing of the (002) plane of $\mathrm{MoS}_{2}$ in the composites slightly increased to $0.62 \mathrm{~nm}$ from $0.61 \mathrm{~nm}$ of the neat $\mathrm{MoS}_{2}$. While the increase is relative low compared with $0.4 \mathrm{~nm}$ of the cellulose molecule thickness, it's probably owing to that some cellulose molecules were intercalated between $\mathrm{MoS}_{2}$ nanosheets to form the "brick-mortar" structure.

\subsection{Thermal stability}

Thermogravimetric (TG) and differential thermogravimetric (DTG) analyses were conducted from 0 to $1000^{\circ} \mathrm{C}$ to study the thermal stability of RC-MoS ${ }_{2}$ nanocomposite films. Figure 4(a) reveals the thermal decomposition process: from room temperature to $100^{\circ} \mathrm{C}$, there is the evaporation of moisture in the films; between $300^{\circ} \mathrm{C}$ and $650^{\circ} \mathrm{C}$, the mass loss occurs because of the cleavage of cellulose molecular chain and carbonization. Moreover, the residual weight of the composites were higher than that of RC, owing to the high thermal stability of $\mathrm{MoS}_{2}$. Figure 4(b) is differential curve of TG curve and indicates the rate of mass loss. The mass loss and decomposition rate of RC-MoS ${ }_{2}$ film decreased after addition of $\mathrm{MoS}_{2}$ nanosheet for the reason that $\mathrm{MoS}_{2}$ has good thermal stability and formed strong hydrogen bond with cellulose. Besides, thermal decomposition temperature of the RC-MoS ${ }_{2}$ composite is a bit higher 
compared with RC. Homogenous dispersion of $\mathrm{MoS}_{2}$ in RC enhances the thermal stability of RC which may inspires the high-temperature application.

\subsection{Mechanical property}

Figure 5 shows the mechanical properties of RC-MoS ${ }_{2}$ films with different $\mathrm{MoS}_{2}$ content. For RC-MoS 1 , the tensile strength (Fig. 5b), Young's Modulus (Fig. 5c) and elongation at break (Fig. 5d) were evidently improved and gradually decreased as the $\mathrm{MoS}_{2}$ content continuously increased. The tensile strength of composites depends much on the interaction between filler and matrix. It increased from $57 \mathrm{MPa}$ to 94 $\mathrm{MPa}$ with the increase of $\mathrm{MoS}_{2}$ content from 0 to $4 \mathrm{wt} \%$, because the strong hydrogen bond formed and stress transfer occurred between cellulose molecules and $\mathrm{MoS}_{2}$ nanosheets. However, when the content of $\mathrm{MoS}_{2}$ continuously increased, there would be more defects, so that the tensile strength decreased. Similarly, moderate addition of $\mathrm{MoS}_{2}$ nanosheets were beneficial for the $\mathrm{MoS}_{2}$ improvement of Young's Modulus and elongation at break as a kind of reinforcing material, but when the content was over $2 \mathrm{wt} \%$, $\mathrm{MoS}_{2}$ nanosheets may aggregate and the films became non-uniform and stress concentration happened, leading to poor mechanical properties.

\subsection{Piezoelectric property}

Piezoelectric constant $\left(d_{33}\right)$ is an important index to characterize the piezoelectric properties; it is the conversion coefficient of transform between mechanical and electric energy. As shown in Fig. 6 , the $d_{33}$ of the neat RC film was $3 \mathrm{pC} / \mathrm{N}$, which reveals that cellulose could be used as a kind of flexible piezoelectric matrix with good advantages of renewability, biodegradability, and biocompatibility. The $d_{33}$ of RC-MoS ${ }_{2}$ films first increased and then decreased with the increased $\mathrm{MoS}_{2}$ content. When the content of $\mathrm{MoS}_{2}$ was $4 \%$, the $d_{33}$ reached the maximum value of $19 \mathrm{pC} / \mathrm{N}$. When the $\mathrm{MoS}_{2}$ content was higher than $4 \%, d_{33}$ decreased probably owing to that the aggregation and stack of $\mathrm{MoS}_{2}$ nanosheet occurred and led to actual lower proportion of $\mathrm{MoS}_{2}$ nanosheet.

To further study the practical application of the RC-MoS 2 piezoelectric films, the nanogenerators were constructed using these piezoelectric films through sandwiched structure. The films were first polarized and then sandwiched between Al foil, which acted as electrode. PI was used as encapsulation layer because it can prevent charge in surrounding environment from interfering the test owing to its insulation property. Figure 7 (a),(b) shows energy harvesting performance of the RC-MoS ${ }_{2}$ nanogenerators with different filler content under a slight press about $0.88 \mathrm{kPa}$. The variation trend of output open-circuit voltage and short-circuit current was consistent with $d_{33}$. The maximum open-circuit voltage was $2 \mathrm{~V}$ and short-circuit current was $150 \mathrm{nA}$, which were 5 and 7.5 times higher than those of the neat RC film, i.e. 0.4 $\mathrm{V}$ and $20 \mathrm{nA}$, respectively. Therefore, the incorporation of $\mathrm{MoS}_{2}$ nanosheets greatly improved the piezoelectric and output properties of the RC film owing to the outstanding piezoelectricity of monolayer or odd-layer $\mathrm{MoS}_{2}$ nanosheets. Fig. S2 shows the "switch polarity" test. When switch the device in the 
forward or reversed mode, the amplitude of output performance is reversed. It indicates that the performance of RC-MoS 2 is generated by piezoelectricity. These flexible RC-MoS 2 nanogenerators are easily fabricated, successfully convert mechanical energy to electric energy, and can be used in complex environment after encapsulation. They have potential applications in the fields of sensors, electronics, and electric skins owing to the good ability of energy harvesting. Besides, these devices have advantages of biocompatibility and biodegradability, which may inspire in-vivo applications.

\section{Conclusion}

In this work, cellulose was dissolved and blended with $\mathrm{MoS}_{2}$ nanosheets in $\mathrm{NaOH} /$ urea aqueous solution to prepare the RC-MoS 2 piezoelectric films and nanogenerators. The structures of the RC-MoS 2 composite films were studied by SEM, FTIR and XRD, and the results indicated the formation of nanolayered structure and strong hydrogen bonding between cellulose molecules and $\mathrm{MoS}_{2}$ nanosheets. Furthermore, the piezoelectric property of the films and their potential applications in energy harvesting were studied in details. The RC-MoS 24 film exhibited a high $d_{33}$ of $19 \mathrm{pC} / \mathrm{N}$, output voltage of $2 \mathrm{~V}$, and current of $150 \mathrm{nA}$ under a slight press, which were $6.3,5$, and 7.5 times higher than those of the RC film, respectively. The incorporation of $\mathrm{MoS}_{2}$ nanosheets thus greatly improved the piezoelectricity and energy harvesting of the $\mathrm{RC}$ film, which could inspire further research. Moreover, the incorporation of $\mathrm{MoS}_{2}$ nanosheets also improved the mechanical properties and thermal stability of the RC film. As a whole, the flexible, renewable, biodegradable, and biocompatible RC-MoS 2 piezoelectric films with high piezoelectricity and output performance are of great significance to environment protection and have potential applications in high-performance energy harvester, wearable sensor, electric skin, etc.

\section{Declarations}

\section{Compliance with ethical standards}

\section{Conflict of interest}

The authors declare that they have no conflict of interest.

\section{Acknowledgements}

This work was supported by the National Natural Science Foundation of China (No. 51703177 and 21704079), the Fundamental Research Funds for the Central Universities (WUT: 2020IB030) and Key laboratory of Processing and Quality Evaluation Technology of Green Plastics of China National Light Industry council, Beijing Technology and Business University, Beijing 100048, China (No.

PQETGP2019007). 


\section{References}

1. Acharya U, Bober P, Trchová M, Zhigunov A, Stejskal J, Pfleger J (2018) Synergistic conductivity increase in polypyrrole/molybdenum disulfide composite. Polymer 150:130-137. http://doi.org/10.1016/j.polymer.2018.07.004

2. Cai J, Zhang $\mathrm{L}$ (2005) Rapid dissolution of cellulose in $\mathrm{LiOH} /$ urea and $\mathrm{NaOH} /$ urea aqueous solutions. Macromol Biosci 5:539-548. http://doi.org/10.1002/mabi.200400222

3. Chen C, Bai Z, Cao Y, Dong M, Jiang K, Zhou Y, Tao Y, Gu S, Gu J, Xu J, Yin X, Xu W (2020) Enhanced piezoelectric performance of $\mathrm{BiCl}_{3}$ /PVDF nanofibers-based nanogenerators. Compos Sci Technol 192: 108100. http://doi.org/10.1016/j.compscitech.2020.108100

4. Chen H, Liu B, Yang Q, Wang S, Liu W, Zheng X. Liu Z, Liu L, Xiong C (2017) Facile one-step exfoliation of large-size 2D materials via simply shearing in triethanolamine. Mater Lett 199:124-127. http://doi.org/10.1016/j.matlet.2017.04.066

5. Chen L, Lv J, Ding L, Yang G, Mao Z, Wang B, Feng X, Zapotoczny S, Sui X (2020) A shape-stable phase change composite prepared from cellulose nanofiber/polypyrrole/polyethylene glycol for electric-thermal energy conversion and storage. Chem Eng J 400:125950. http://doi.org/10.1016/j.cej.2020.125950

6. Fan FR, Tang W, Wang ZL (2016) Flexible nanogenerators for energy harvesting and self-powered electronics. Adv Mater 28:4283-4305. http://doi.org/10.1002/adma.201504299

7. Fang LS, Tsai CY, Xu MH, Wu SW, Lo WC, Lu YH, Fuh YK (2019) Hybrid nano-textured nanogenerator and self-powered sensor for on-skin triggered biomechanical motions. Nanotechnol 31:155502. http://doi.org/10.1088/1361-6528/ab6677

8. French AD (2014) Idealized powder diffraction patterns for cellulose polymorphs. Cellulose 21:885896. https://doi.org/10.1007/s10570-013-0030-4

9. French AD (2020) Increment in evolution of cellulose crystallinity analysis. Cellulose 27:5445-5448. https://doi.org/10.1007/s10570-020-03172-z

10. Goldemberg J (2007) Ethanol for a sustainable energy future. Science 315:808-810. http://doi.org/10.1126/science.1137013

11. Isobe N, Kim UJ, Kimura S, Wada M, Kuga S (2011) Internal surface polarity of regenerated cellulose gel depends on the species used as coagulant. J Colloid Interface Sci 359: 194-201. http://dx.doi.org/10.1016/j.jcis.2011.03.038

12. Kim JH, Mun S, Ko HU, Yun GY, Kim J (2014) Disposable chemical sensors and biosensors made on cellulose paper. Nanotechnol 25:092001.

13. http://doi.org/10.1126/science. 1137013

14. Klemm D, Philpp B, Heinze T, Heinze U, Wagenknecht W (1998) Comprehensive cellulose chemistry: Fundamentals and analytical methods. Wiley-VCH, Weinheim, pp 377

15. Koka, A, Sodano HA (2014) A low-frequency energy harvester from ultralong, vertically aligned $\mathrm{BaTiO}_{3}$ nanowire arrays. Adv Energy Mater 11:1301660. 
http://doi.org/4:1301660.10.1002/aenm.201301660

16. Krishnamoorthy K, Pazhamalai P, Veerasubramani GK, Kim SJ (2016) Mechanically delaminated few layered $\mathrm{MoS}_{2}$ nanosheets based high performance wire type solid-state symmetric supercapacitors. J Power Sources 321:112-119. http://doi.org/10.1016/j.jpowsour.2016.04.116

17. Li Y, Xu M, Xia Y, Wu J, Sun X, Wang S, Hu GH, Xiong CX (2020) Multilayer assembly of electrospun/electrosprayed PVDF-based nanofibers and beads with enhanced piezoelectricity and high sensitivity. Chem Eng J 388:124205. http://doi.org/10.1016/j.cej.2020.124205

18. Lovinger AJ (1983) Ferroelectric polymers, Science 220:1115-1121. http://doi.org/10.1126/science.220.4602.1115

19. Lv X, Wu J, Zhang X (2020) A new concept to enhance piezoelectricity and temperature stability in KNN ceramics. Chem Eng J 402:126215. http://doi.org/10.1016/j.cej.2020.126215

20. MiShra S, Unnikrishnan L, Nayak SK, Mohanty S (2019) Advances in piezoelectric polymer composites for energy harvesting applications: a systematic review. Macromol Mater Eng 304:18800463. https://doi.org/10.1002/mame.201800463

21. Niu L, Coleman JN, Zhang H, Shin H, Chhowalla M, Zheng Z (2016) Production of two-dimensional nanomaterials via liquid-based direct exfoliation. Small 122:272-293.

https://doi.org/10.1002/smll.201502207

22. Pandey JK, Ahn SH, Lee CS,Mohanty AK, Misra M (2010) Recent advances in the application of natural fiber based composites. Macromol Mater Eng 295: 975-989. https://doi.org/10.1002/mame.201000095

23. Park DY, Joe DJ, Kim DH, Park H, Han JY, Jeong CK, Park H, Park JG, Joung B, Lee KJ

24. (2020) Self-powered real-time arterial pulse monitoring using ultrathin epidermal piezoelectric sensors. Adv Mater 29:1702308. https://doi.org/10.1002/adma.201702308

25. Park K, Bae SB, Yang SH, Lee HI, Lee K, Lee SJ (2014) Lead-free $\mathrm{BaTiO}_{3}$ nanowires-based flexible nanocomposite generator. Nanoscale 6:8962-8968. https://doi.org/10.1039/c4nr02246g

26. Qi H, Chang C, Zhang L (2009) Properties and applications of biodegradable transparent and photoluminescent cellulose films prepared via a green process. Green Chem 11:177-184. https://doi.org/10.1039/B814721C

27. Raghunathan SP, Narayanan S, Poulose AC, Joseph R (2017) Flexible regenerated cellulose/polypyrrole composite films with enhanced dielectric properties. Carbohydr Polym 157:1024-1032. https://doi.org/10.1016/j.carbpol.2016.10.065

28. Sabo R, Yermakov A, Law CT, Elhajjar R (2016) Nanocellulose-enabled electronics, energy harvesting devices, smart materials and sensors: A review. J Renewable Mater 4:297-312. https://doi.org/10.7569/JRM.2016.634114

29. Sahatiya P, Kannan S, Badhulika S. Few layer $\mathrm{MoS}_{2}$ and in situ poled PVDF nanofibers on low cost paper substrate as high performance piezo-triboelectric hybrid nanogenerator: Energy harvesting 
from handwriting and human touch. Applied Materialstoday 13:91-99.

https://doi.org/10.1016/j.apmt.2018.08.009

30. Sappati KK, Bhadra S (2018) Piezoelectric polymer and paper substrates: A review. Sensors 18:3605. https://doi.org/10.3390/s18113605

31. Shi K, Huang X, Sun B, Wu Z, He J, Jiang P (2019) Cellulose/BaTiO 3 aerogel paper based flexible piezoelectric nanogenerators and the electric coupling with triboelectricity. Nano Energy 57: 450-458. https://doi.org/10.1016/j.nanoen.2018.12.076

32. Sohn A, Choi S, Han SA, Kim TH, Kim Y, Kim SW (2019) Temperature-dependent piezotronic effect of $\mathrm{MoS}_{2}$ monolayer. Nano Energy 58:811-816. https://doi.org/10.1016/j.nanoen.2019.02.017

33. Song Y, Shi Z, Hu G-H, Xiong C, Isogai A, Yang Q (2021) Recent advances in cellulose-based piezoelectric and triboelectric nanogenerators for energy harvesting: a review. J Mater Chem $A$ https://doi.org/10.1039/D0TA08642H

34. Tayeb P, Tayeb AH (2019) Application of nanocellulose in sustainable electrochemical and piezoelectric systems: A review. Carbohydr Polym 224:115149. https://doi.org/10.1016/j.carbpol.2019.115149

35. Toroń B, Szperlich T, Nowak M Stro'z D, Rzychon T (2018) Novel piezoelectric paper based on SbSI nanowires. Cellulose 25: 7-15. https://doi.org/10.1007/s10570-017-1597-y

36. Vivekananthan V, Purusothaman Y, Chrasekhar S, Kim SJ (2018) Biocatible collagen-nanofibrils: An approach for sustainable energy harvesting and battery-free humidity sensor applications. ACS Appl. Mater Interfaces 10: 18650-18656. https://doi.org/10.1021/acsami.8b02915

37. Wang J, Chen H, Li X, Zhang C, Yu W, Zhou L, Yang Q, Shi Z, Xiong C (2020) Flexible dielectric film with high energy density based on chitin/boron nitride nanosheets. Chem Eng J 383: 123147. https://doi.org/10.1016/j.cej.2019.123147

38. Wang ZL, Song J (2016) Piezoelectric nanogenerators based on zinc oxide nanowire arrays. Science 312:214-246. https://doi.org/10.1126/science.1124005

39. Wang C, Yang Z, Wang X, Yu Q (2019) New research progress of functional wood. J For Eng 4(03):10-18. https://doi.org/10.13360/j.issn.2096-1359.2019.03.002

40. Wang ZL, Wu W (2012) Nanotechnology-enabled energy harvesting for self-powered micro-/nanosystems. Angew Chem 51:11700-11721. https://doi.org/10.1002/anie.201201656

41. Wei $H$, Wang $H$, Xia Y, Cui D, Shi Y, Dong M, Liu C, Ding T, Zhang J, Ma Y, Wang N, Wang Z, Sun Y, Wei R, Guo Z (2018) An overview of lead-free piezoelectric materials and devices. J Mater Chem 6:1244612467. https://doi.org/10.1039/C8TC04515A

42. Wu T, Song Y, Shi Z, Liu D, Chen S, Xiong C, Yang Q (2021) High-performance nanogenerators based on flexible cellulose nanofibril/MoS 2 nanosheet composite piezoelectric films for energy harvesting. Nano Energy 80:105541. https://doi.org/10.1016/j.nanoen.2020.105541

43. Wu Y, Qu J, Daoud WA, Wang L, Qi T (2019) Flexible composite-nanofiber based piezo-triboelectric nanogenerators for wearable electronics. J Mater Chem A 7: 13347-13355. 
https://doi.org/10.1039/C9TA02345C

44. Xu S, Qin Y, Xu C, Wei Y, Yang R, Wang ZL (2010) Self-powered nanowire devices. Nat Nanotech 5:366-373. https://doi.org/10.1038/nnano.2010.46

45. Yang D, Qiu Y, Wang T, Song W, Wang Z, Xu J, Feng Q, Zong Y, Sun X (2016) Growth of 3D branched ZnO nanowire for DC-type piezoelectric nanogenerators. J Mater Sci Mater Electron 7:6708-6712. https://doi.org/10.1007/s10854-016-4619-x

46. Yang J, Xie H, Chen H, Shi Z, Wu T, Yang Q, Xiong C (2018) Cellulose nanofibril/boron nitride nanosheet composites with enhanced energy density and thermal stability by interfibrillar crosslinking through $\mathrm{Ca}^{2+}$. J Mater Chem 6:1403-1411. https://doi.org/10.1039/c7ta08188j

47. Yang L, Zhao Q, Chen K, Ma Y, Wu Y, Ji H, Qiu J (2020) PVDF-based composition-Gradient multilayered nanocomposites for flexible high-performance piezoelectric nanogenerators. ACS Appl Mater Interfaces 12:11045-11054. https://doi.org/10.1021/acsami.9b23480

48. Yang Q, Saito T, Berglund L, Isogai A (2015) Cellulose nanofibrils improve the properties of allcellulose composites by the nano-reinforcement mechanism and nanofibrilinduced crystallization. Nanoscale 7(42):17957-17963. https://doi.org/10.1039/c5nr05511c

49. Ye S, Cheng C, Chen X, Shao J, Zhang J, Hu H, Tian H, Li X, Ma L, Jia W (2019) High-performance piezoelectric nanogenerator based on microstructured $\mathrm{P}(\mathrm{VDF}-\mathrm{TrFE}) / \mathrm{BNNTs}$ composite for energy harvesting and radiation protection in space. Nano Energy 60:701-714. https://doi.org/10.1016/j.nanoen.2019.03.096

50. Yin Y, Zhang C, Yu W, Kang G, Yang Q, Shi Z, Xiong C (2020) Transparent and flexible cellulose dielectric films with high breakdown strength and energy density. Energy Storage Mater 26:105-111. https://doi.org/10.1016/j.ensm.2019.12.034

51. Zhai L, Mun s, Gao X, Kim JW, Kim J (2015) Cellulose electro-active paper fabricated by facile solvent exchange pretreatment and its physical and electromechanical properties. Cellulose 22:927-933. https://doi.org/10.1007/s10570-015-0553-y

52. Zhang H, Wang Z, Zhang Z, Wu J, Zhang J, He J (2007) Regenerated-cellulose/multiwalled-carbonnanotube composite fibers with enhanced mechanical properties prepared with the ionic liquid 1allyl-3-methylimidazolium chloride. Adv Mater 5:698-704. https://doi.org/10.1002/adma.200600442

53. Zhao F, Zhang C, Liu J, Liu L, Cao X, Chen X, Lei B, Shao L (2020) Periosteum structure/functionmimicking bioactive scaffolds with piezoelec-tric/chem/nano signals for critical-sized bone regeneration. Chem Eng J 402:126203. https://doi.org/10.1016/j.cej.2020.126203

54. Zhao Q, Lu Y, Chen K, Ma Y, Peng Q, Ji H, Qiu J (2020) Flexible textured $\mathrm{MnO}_{2}$ nanorods/ PVDF hybrid films with superior piezoelectric performance for energy harvesting application. Compos Sci Technol 199:108330. https://doi.org/10.1016/j.compscitech.2020.108330

55. Zhou Y, Liu W, Huang X, Zhang A, Zhang Y, Wang ZL (2016) Theoretical study on two-dimensional $\mathrm{MoS}_{2}$ piezoelectric nanogenerators. Nano Res 9:800-807. https://doi.org/10.1007/s12274-015-09598 


\section{Figures}

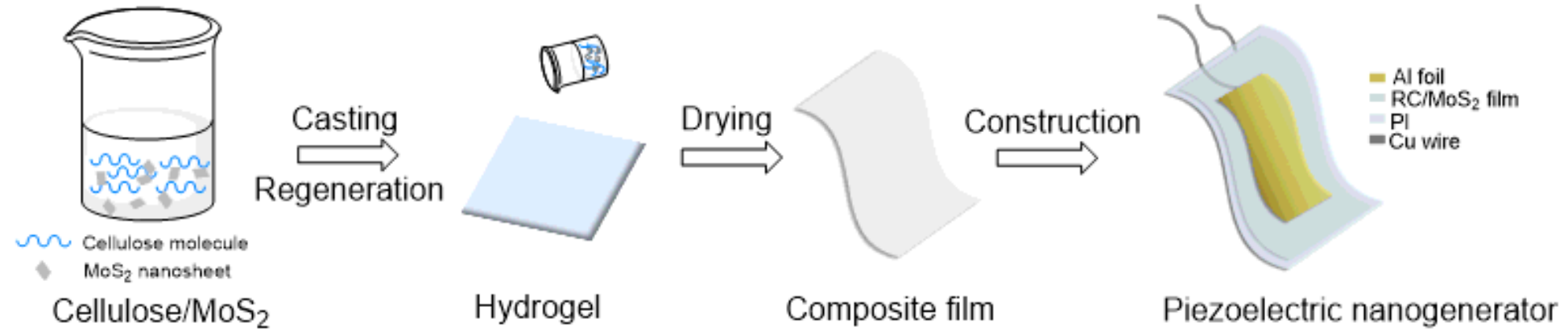

nanosheet dispersion

Figure 1

Schematic of fabrication process for the RC/MoS2 nanogenerator
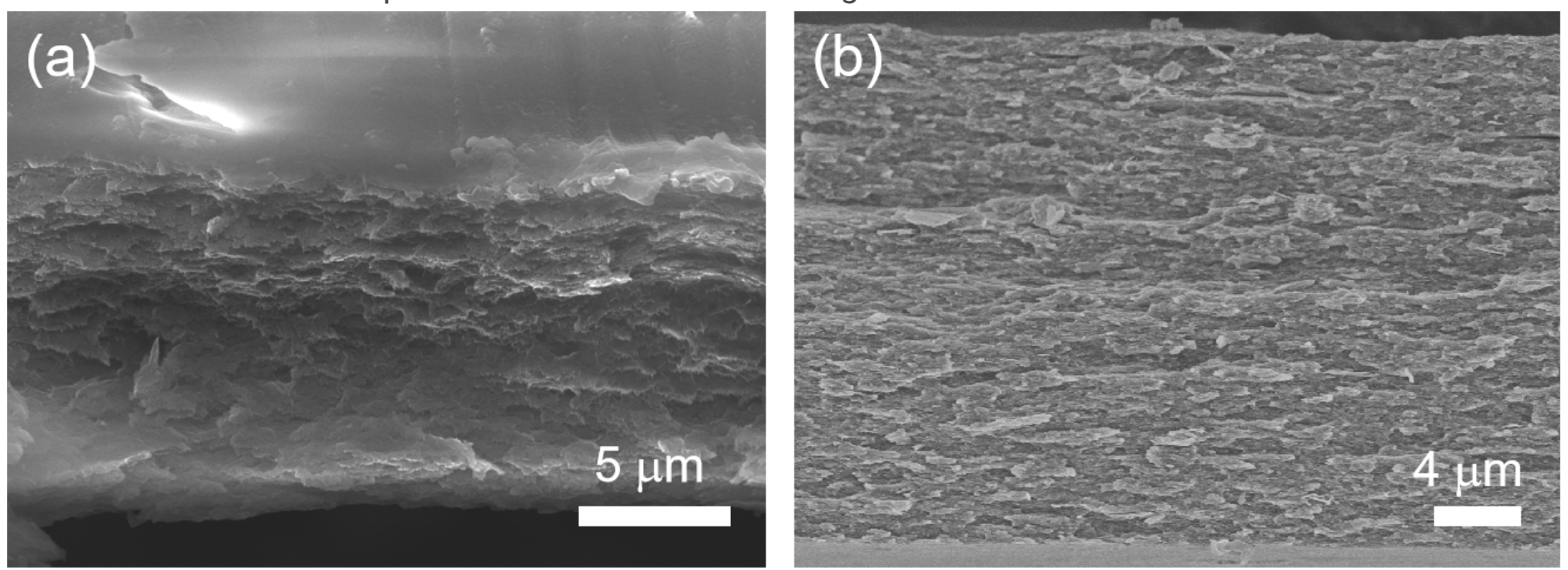

(c)

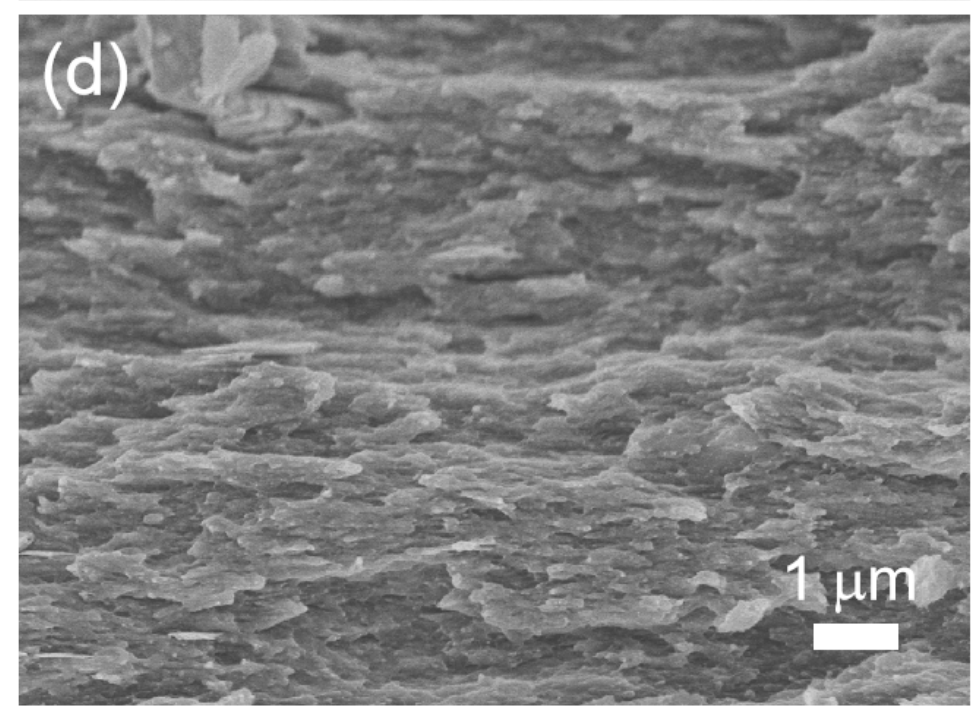

Figure 2

SEM images of cross-sections of the RC (a, b) and RC-MoS24 (c, d) films. 


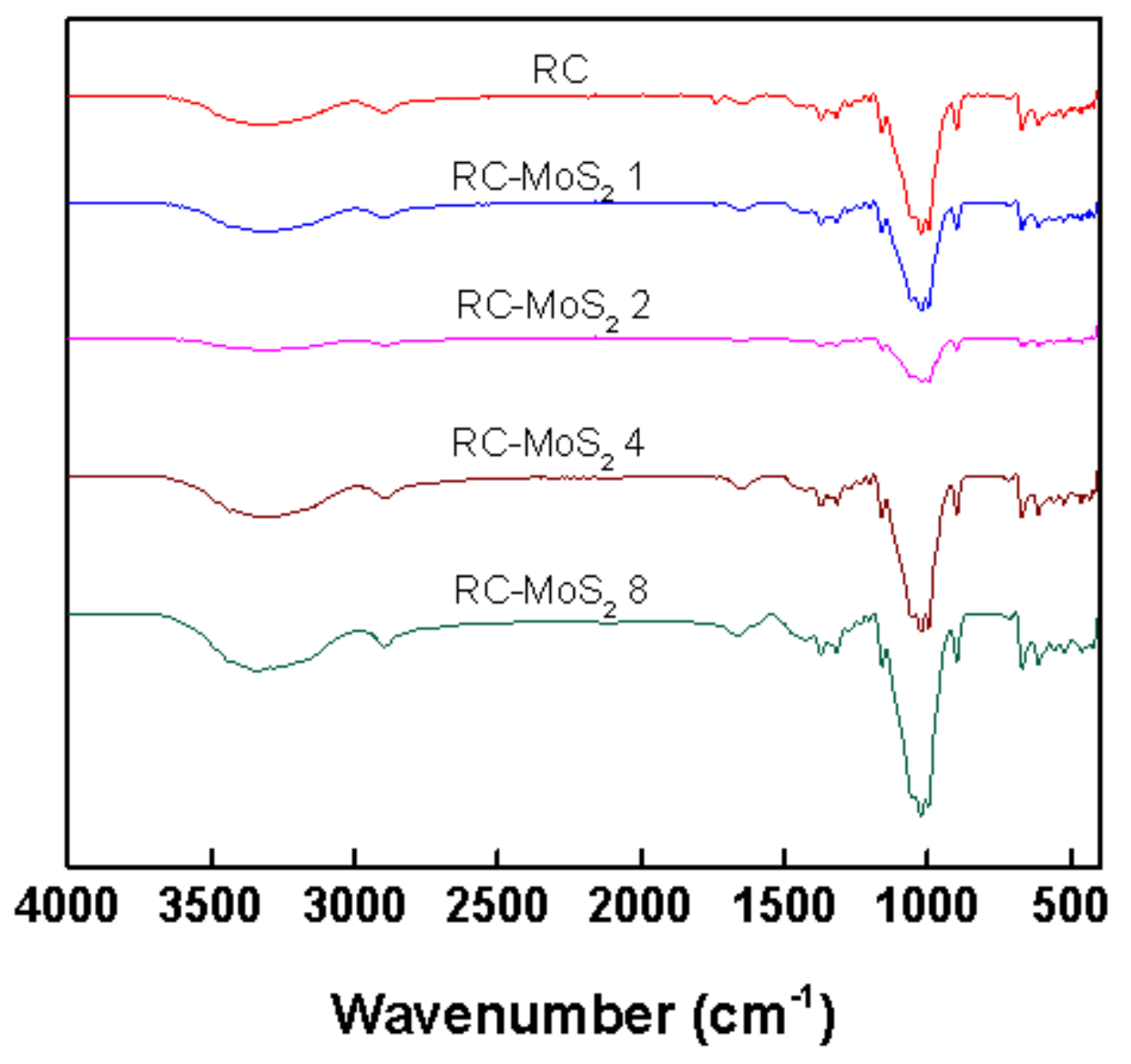

Figure 3

FTIR spectra of RC and RC-MoS2 films.
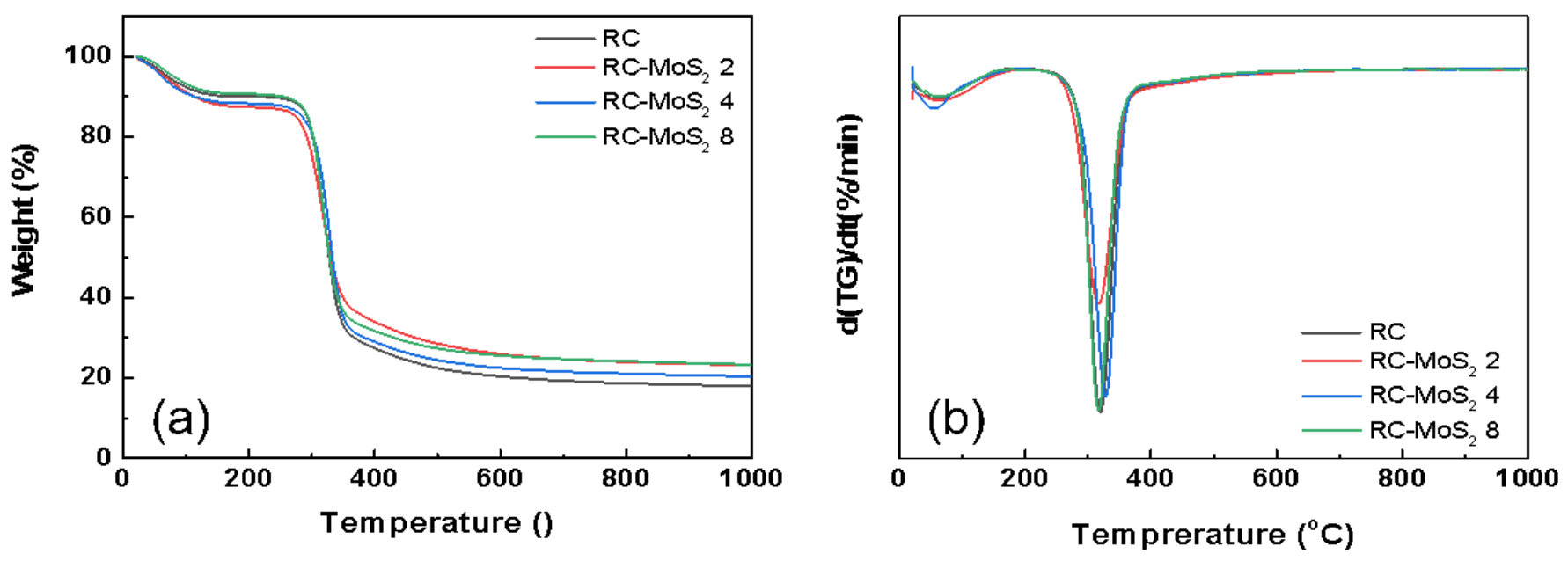

Figure 4

TG (a) and DTG (b) curves of RC and RC-MoS2 films. 

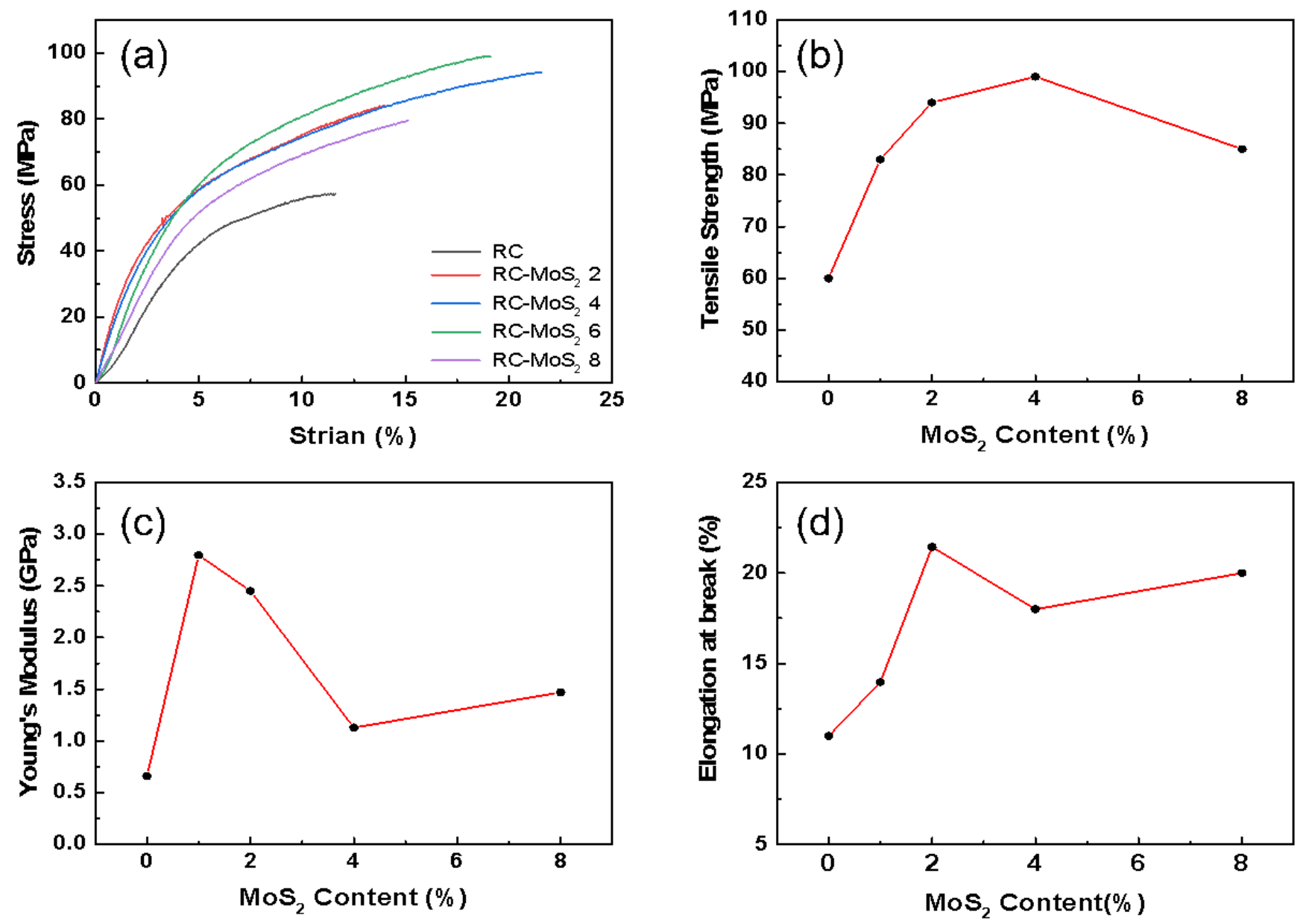

Figure 5

Stress-Strain (a), Tensile strength (b), Young's Modulus (c) and elongation at break (d) of the RC and RCMoS2 composite films with different MoS2 content. 


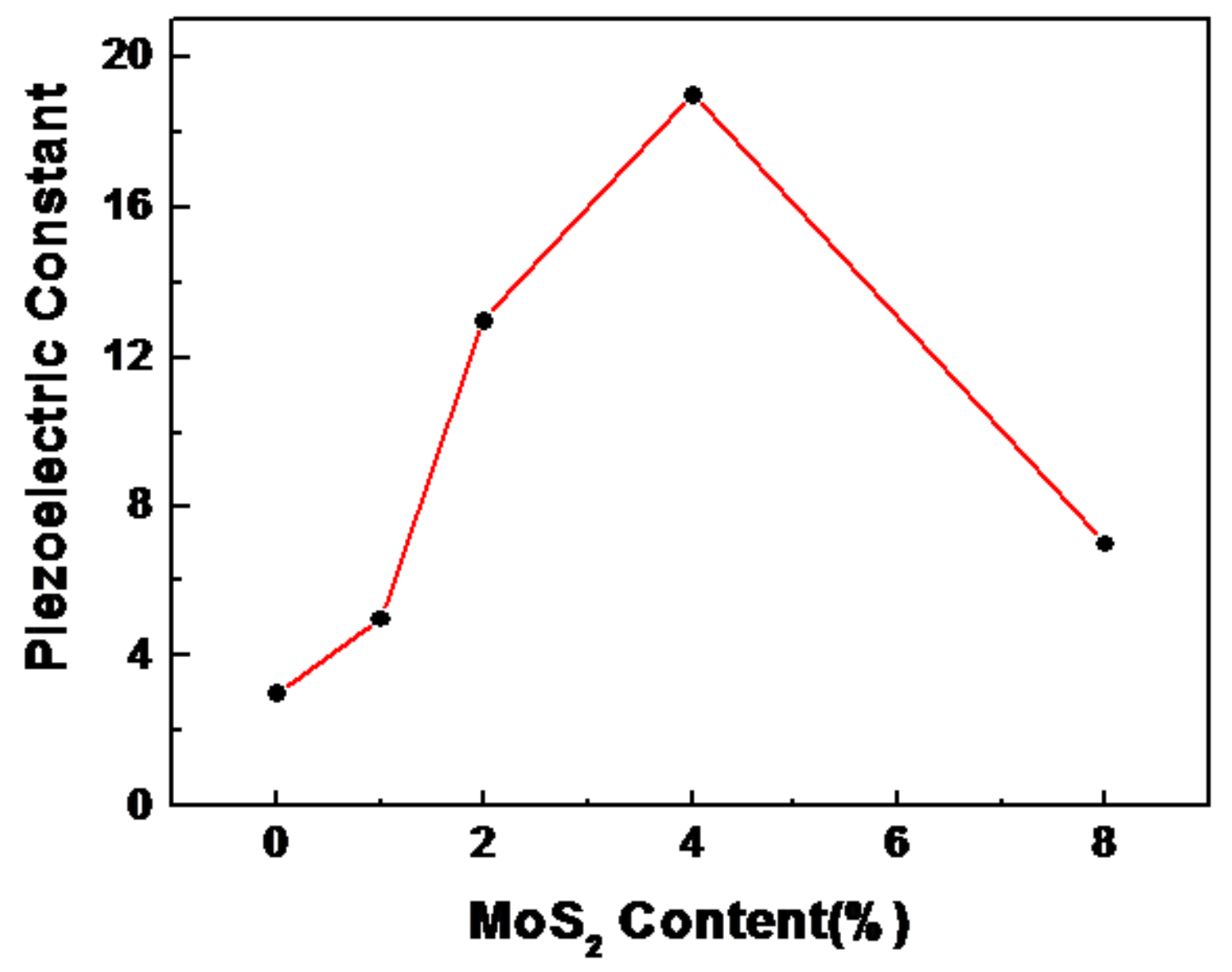

Figure 6

Effects of MoS2 contents on the piezoelectric constants (d33) of RC-MoS2 films.
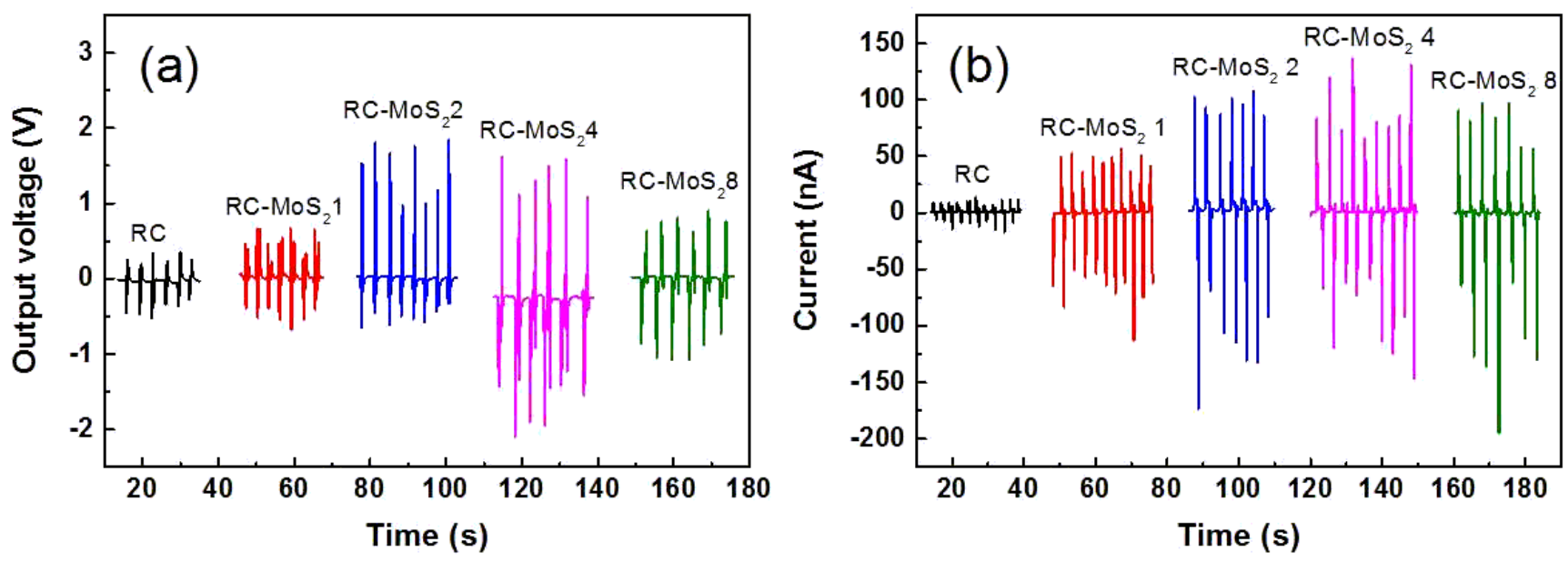

Figure 7 
The open-circuit voltage (a) and short-circuit current (b) Piezoelectric voltage of the RC-MoS2 4 based PENG with forward and reversed connection modes of the RC and RC-MoS2 composite films.

\section{Supplementary Files}

This is a list of supplementary files associated with this preprint. Click to download.

- RChenSLsupplementaryinformation.doc 